\section{Vertragsarztrechtliche Anforderungen an die Auslagerung radiologischer Leistungen}

\section{Auslagerung radiologischer Leistungen und persönliche Leistungserbringung}

Im Rahmen der Auslagerung radiologischer Leistungen ergeben sich verschiedene, von der Beantwortung vertragsarztrechtlicher Vorgaben abhängige Fragestellungen.

Einerseits stellt sich die Frage, ob eine ärztliche Leistung dergestalt ausgelagert werden kann, dass z. B. die Untersuchung des Patienten durch einen Arzt am Standort der Praxis und die Befundung durch einen anderen Arzt an einem anderen Ort, auch aus aktuellem Anlass der SARS-CoV-2-Pandemie beispielsweise im Homeoffice, erfolgen kann.

Für die Beantwortung dieser Frage ist vornehmlich der Grundsatz der persönlichen Leistungserbringung relevant. Dieser verlangt, dass eine ärztliche Leistung einheitlich von ein und demselben Arzt erbracht wird. Gleichwohl gibt es von diesem Grundsatz Ausnahmen: Diese haben wir bereits in unserem zweiteiligen Beitrag „Grundsatz der persönlichen Leistungserbringung in der vertragsärztlichen Versorgung, insbesondere bei kooperativer Zusammenarbeit“ (RöFo 06/2019, S. 580-584 und RöFo 07/ 2019, S. 672-676) ausführlich dargestellt.

Eine andere Frage ist, ob eine radiologische Leistung - ob nun gänzlich oder nur teilweise, ggfs. auch durch einen anderen Arzt überhaupt an einen anderen Standort ausgelagert werden darf und welche vertragsarztrechtlichen Anforderungen hierbei zu beachten sind. Der Beantwortung dieser Fragestellung widmet sich der vorliegende Beitrag.

\section{Grundsatz: Leistungserbrin- gung am Praxisstandort}

So wie der Grundsatz der persönlichen Leistungserbringung davon ausgeht, dass ein und derselbe Arzt die in einer Gebührenordnungsposition (GOP) des Einheitlichen Bewertungsmaßstabs (EBM) beschriebene
Leistung ausführt, geht das ärztliche Berufsrecht von dem Grundsatz aus, dass der Arzt seine Leistungen am Praxisstandort erbringt.

$\S 17$ Abs. 1 der Musterberufsordnung (MBO-Ä) bindet die Ausübung ambulanter ärztlicher Tätigkeit außerhalb von Krankenhäusern einschließlich konzessionierter Privatkliniken an die Niederlassung in einer Praxis (Praxissitz). Im Vertragsarztrecht findet sich dieser Grundsatz in $\$ 24$ Abs. 1 Zulassungsverordnung für Vertragsärzte (Ärzte-ZV): Die Zulassung erfolgt für den Ort der Niederlassung als Arzt, dem Vertragsarztsitz.

Dies schließt gleichwohl die Auslagerung ärztlicher Leistungen nicht aus, vgl. §17 Abs. 1 MBO-Ä: Durch gesetzliche Vorschriften kann von dem Grundsatz abgewichen werden, dass der Arzt seine Leistungen am Praxissitz zu erbringen hat. Die Auslagerung von Leistungen bleibt aber die Ausnahme, sie setzt eine Rechtsgrundlage und eine Genehmigung voraus, allerdings nicht für den ausgelagerten Praxisteil, dieser ist nur anzeigepflichtig. Wer also ohne Rechtsgrundlage und/oder ggfs. ohne Genehmigung Leistungen auslagert, verstößt gegen vertragsarztrechtliche Regelungen, riskiert den Verlust der Vergütung für die ausgelagerten Leistungen und möglicherweise die Einleitung eines Disziplinarverfahrens.

\section{Ausnahmen}

\section{a) Auslagerung von Leistungen in einen ausgelagerten Praxisteil}

Zunächst erlaubt § 24 Abs. 5 Ärzte-ZV, Leistungen in einem ausgelagerten Praxisteil zu erbringen.

„Erbringt der Vertragsarzt spezielle Untersuchungs- und Behandlungsleistungen an weiteren Orten in räumlicher Nähe zum Vertragsarztsitz (ausgelagerte Praxisräume), hat er Ort und Zeitpunkt der Aufnahme der Tätigkeit seiner Kassenärztlichen Vereinigung unverzüglich anzuzeigen. “

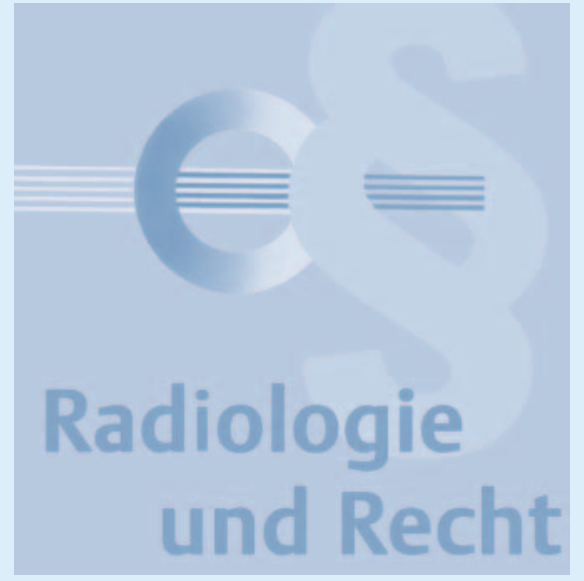

Will ein Radiologe außerhalb seiner Praxisräume beispielsweise ein PET-CT betreiben, so ist dieses Vorhaben nach der vorstehenden Regelung zulässig. Es handelt sich um eine spezielle Untersuchungsleistung, die an einem weiteren Ort erbracht werden soll. Allerdings sind folgende Einschränkungen zu beachten:

- Ein ausgelagerter Praxisteil darf keine Anmeldung enthalten.

- In einem ausgelagerten Praxisteil dürfen keine Termine mit dem Patienten abgestimmt oder Verwaltungsaufgaben der Praxis erledigt werden.

- In einem ausgelagerten Praxisteil darf keine Sprechstunde eines Arztes stattfinden.

- In einem ausgelagerten Praxisteil dürfen nur die ausgelagerten Leistungen erbracht werden. Diese dürfen dabei die Leistungen am Praxisstandort nicht überwiegen.

Hinsichtlich der Erbringung von Leistungen in einem ausgelagerten Praxisteil besteht grundsätzlich keine Genehmigungs-, sondern einzig eine Anzeigepflicht gegenüber der zuständigen Kassenärztlichen Vereinigung (KV). Die Leistungen dürfen erbracht und abgerechnet werden, sobald die Anzeige bei der zuständigen KV eingegangen ist. Allerdings darf der Arzt im Zuge des Anzeigeverfahrens ein Schweigen der KV auf seine Anzeige nicht als Bestätigung der Rechtmäßigkeit der Leistungserbringung im ausgelagerten Praxisteil missverstehen. Vielmehr obliegt es der Verantwortung des Arztes, sicherzustellen, dass die Voraussetzungen für den Betrieb des ausgelagerten Praxisteils auch wirklich vorliegen. Hierzu gehört auch, dass eventuell erforderliche 
Genehmigungen nach den Qualitätssicherungsvereinbarungen eingeholt wurden. Sofern hinsichtlich des Vorliegens dieser Voraussetzungen Zweifel bestehen, sollte, auch im Hinblick auf ggfs. drohende Regresse im Falle des Nichtvorliegens der Voraussetzungen zur Leistungserbringung im ausgelagerten Praxisteil, eine schriftliche Zusage der zuständigen KV eingeholt werden.

In der Praxis wirft die Regelung des § 24 Abs. 5 Ärzte-ZV jedoch Fragen auf, die wegen ihrer Bedeutung für die Leistungserbringung im Fachgebiet Radiologie nachstehend vertieft beantwortet werden sollen:

- Bedeutet das Wort „spezielle Untersuchungs- und Behandlungsleistungen“, dass es sich nur um solche handeln darf, die am eigentlichen Praxisstandort nicht erbracht werden?

Der Begriff der speziellen Untersuchungsund Behandlungsleistung ist als Abgrenzung zu den gesamten Untersuchungsund Behandlungsleistungen zu verstehen, wie sie in der Arztpraxis erbracht werden. Das Tatbestandsmerkmal soll sicherstellen, dass der ausgelagerte Praxisteil nicht wie eine Arztpraxis betrieben wird, sondern dort ausschließlich einzelne Leistungen durchgeführt werden. Daher können in einem ausgelagerten Praxisteil MRT-Leistungen angeboten werden, auch wenn sich am Praxisstandort bereits ein MRT befindet.

- Handelt es sich auch bei den digitalen Bildbefundungen eines Radiologen, die dieser während der COVID-19-Pandemie in seinem Homeoffice erbringt, um „spezielle Untersuchungsleistungen“?

Fraglich ist, ob es sich bei dem Homeoffice überhaupt um einen ausgelagerten Praxisteil handelt. Ein ausgelagerter Praxisteil ist ein Raum, der einer Praxis zugeordnet ist, ohne sich am Standort der Praxis zu befinden. Das Homeoffice ist jedoch kein abgegrenzter Raum, der der Praxis zugeordnet ist, sondern kann je nach räumlicher Verfügbarkeit wechseln. Für die Sicherheit der Leistungserbringung entscheidend ist beim Homeoffice auch nicht die räumliche $\mathrm{Zu}$ ordnung - abgesehen davon, dass Dritte auf das Endgerät/die dort vorgehaltenen
Daten des Arztes keinen Zugriff haben dürfen -, sondern die Sicherheit der Datenverbindung. Es spricht daher einiges dafür, dass das Homeoffice keinen ausgelagerten Praxisteil darstellt, sondern die Tätigkeit im Homeoffice einer Tätigkeit am Vertragsarztsitz gleichgestellt ist.

Hierfür sprechen auch die Vereinbarung über die Anforderungen an die technischen Verfahren zur Videosprechstunde gemäß $\S 291 \mathrm{~g}$ Abs. 4 SGB V vom 21. Oktober 2016 in der Fassung vom 27. Juli 2020 und die Vereinbarung über die Anforderungen an die technischen Verfahren zur telemedizinischen Erbringung der konsiliarischen Befundbeurteilung von Röntgenaufnahmen in der vertragsärztlichen Versorgung gemäß §291 g Abs. 1 S. 1 SGB V mit Wirkung zum 1.7.2016. Diese definieren die Anforderungen an die datentechnischen Voraussetzungen, stellen jedoch keine speziellen Anforderungen an die Räume, abgesehen von der Vorgabe, dass die Videosprechstunde zur Gewährleistung der Datensicherheit sowie eines störungsfreien Ablaufes in geschlossenen Räumen, die eine angemessene Privatsphäre sicherstellen, stattzufinden hat.

\section{- Was ist unter räumlicher Nähe zum Vertragsarztsitz zu verstehen?}

§24 Abs. 5 Ärzte-ZV geht davon aus, dass der Vertragsarzt selbst Leistungen im ausgelagerten Praxisteil erbringt. Dann muss aber sichergestellt sein, dass er zur Erfüllung seiner vertragsärztlichen Pflichten den Vertragsarztsitz in angemessener Zeit erreichen kann. Insofern könnte auf die Rechtsprechung zurückgegriffen werden, die für die Entfernung zwischen Belegklinik und Vertragsarztsitz/Wohnsitz des Belegarztes auf eine Fahrtzeit von maximal 30 Minuten abstellt. Bei arbeitsteiliger Leistungserbringung, z. B. in einer Berufsausübungsgemeinschaft (BAG), verliert dieses Tatbestandsmerkmal jedoch an Bedeutung, wenn die Leistungserbringung am Praxisstandort durch die Anwesenheit anderer Ärzte der BAG gewährleistet ist. Dies kommt darin zum Ausdruck, dass die Regelungen zur überörtlichen Berufsausübungsgemeinschaft (ÜBAG) keine räumliche Begrenzung für die Entfernungen zwischen deren Standorten vorsehen.

\section{b) Auslagerung von Leistungen in eine Nebenbetriebsstätte}

Von dem ausgelagerten Praxisteil ist die Nebenbetriebsstätte (auch synonym: Zweigpraxis) zu unterscheiden. Während in einem ausgelagerten Praxisteil keine Sprechstunde abgehalten werden darf, sondern nur spezielle Untersuchungs- oder Behandlungsleistungen erbracht werden dürfen, inkludiert der Betrieb einer Nebenbetriebsstätte auch die ärztliche Sprechstunde. Es handelt sich also um einen weiteren vollwertigen (Zweig-) Praxisstandort.

Nach § 24 Abs. 3 Ärzte-ZV benötigt der Vertragsarzt für eine Nebenbetriebsstätte, die Genehmigung des Vorstandes der für den KV-Bezirk zuständigen Kassenärztlichen Vereinigung. Befindet sich die Nebenbetriebsstätte in einem anderen KV-Bezirk als die Hauptbetriebsstätte, so benötigt der Vertragsarzt für den Betrieb der Nebenbetriebsstätte die Ermächtigung des Zulassungsausschusses, der für den KV-Bezirk zuständig ist, in dem die Nebenbetriebsstätte betrieben werden soll.

Die Genehmigung/Ermächtigung zum Betrieb einer Nebenbetriebsstätte setzt voraus, dass

- einerseits hierdurch die Versorgung der Versicherten am Standort der Nebenbetriebsstätte verbessert wird und

- andererseits die ordnungsgemäße Versorgung der Versicherten am Ort des Vertragsarztsitzes nicht beeinträchtigt wird.

In der Praxis bereitet insbesondere der Nachweis der Verbesserung der Versorgung der Versicherten am Standort der Nebenbetriebsstätte Schwierigkeiten:

Zwar kann nach §24 Abs. 3 S. 4 Ärzte-ZV eine Verbesserung der Versorgung auch darin bestehen, dass eine bestehende Praxis am Vertragsarztsitz als Zweigpraxis fortgeführt wird. Wer also eine Praxis erwerben und diese unter Verzicht des Verkäufers zum Zweck der Anstellung (in dessen bisheriger Praxis) als Zweigpraxis fortführen will, verbessert durch die Fortführung der bisherigen Praxis als Zweigpraxis die Versorgung der Versicherten. 
Im Übrigen begibt sich der Vertragsarzt, der eine Zweigpraxis betreiben will, auf rechtliches „Glatteis“.

Erforderlich - aber auch ausreichend - ist nach dem Urteil des Bundessozialgerichts vom 16.12.2015 (Az. B 6 KA 37/14 R), dass das bestehende Leistungsangebot an dem Ort, an dem die Zweigpraxis betrieben werden soll, zum Vorteil für die Versicherten in qualitativer oder quantitativer Hinsicht erweitert wird. Eine solche Verbesserung kann etwa dann gegeben sein, wenn der in der Zweigpraxis tätige Vertragsarzt im Vergleich zu den bereits vor Ort tätigen Ärzten über andere oder weitergehende qualifikationsgebundene Genehmigungen nach $\S 135$ Abs. 2 SGB V verfügt, ein differenzierteres Leistungsspektrum anbietet oder wenn er eine besondere Untersuchungsoder Behandlungsmethode anwenden kann, die etwa besonders schonend ist oder bessere Diagnoseergebnisse liefert. Darauf, wie viele Patienten das Angebot nutzen können, kommt es nicht an. Es geht - anders als bei der Sonderbedarfszulassung - nicht um die Feststellung eines Bedarfs. Mit anderen Worten: Die Verbesserung der Versorgung setzt nicht voraus, dass die Zweigpraxis erforderlich ist. Ebenso verbietet sich damit eine Heranziehung der - namentlich zur Sonderbedarfszulassung ergangenen - Rechtsprechung, wonach es Patienten bei speziellen Leistungen zuzumuten ist, längere Wege in Kauf zu nehmen. Hieraus ließe sich schließen, dass die Hürden für die Genehmigung/Ermächtigung zum Betrieb einer Zweigpraxis nicht all zu hoch sind.

Problematisch wird die Erbringung des Nachweises jedoch dann, wenn in der Nähe des Standorts der Nebenbetriebsstätte Praxen mit vergleichbarem Leistungsangebot existieren. So hat das Sozialgericht Marburg mit Urteil vom 17.06.2015 (Az. S 16 KA 460/12) entschieden, dass eine Zweigpraxis mit dem Angebot radiologischer Diagnostik, Computertomographie und konventioneller Röntgenleistungen die Versorgung nicht verbessert, wenn in einer Entfernung von unter 17 Kilometern bereits zwei bestehende radiologische Praxen jeweils das vollständige radiologische Leistungsangebot abbilden.
Bei dem Tatbestandsmerkmal der „Verbesserung der Versorgung der Versicherten“ handelt es sich um einen sogenannten unbestimmten Rechtsbegriff. Dies führt in der Praxis zu Schwierigkeiten, weil sich aufgrund dessen nicht mit der für Investitionsentscheidungen erforderlichen Sicherheit prognostizieren lässt, ob mit einer Genehmigung/Ermächtigung zum Betrieb der Zweigpraxis gerechnet werden kann. Diese Unsicherheit wird dadurch verstärkt, dass anders als bei der Auslagerung von Leistungen in einen ausgelagerten Praxisteil - gegen die Genehmigung/Ermächtigung zum Betrieb einer Zweigpraxis ein sogenannter Drittwiderspruch, d. h. der Widerspruch einer konkurrierenden Praxis, zulässig ist. Auch die Genehmigung/Ermächtigung zum Betrieb einer Nebenbetriebsstätte ist also noch keine hinreichende Stütze für eine Investitionsentscheidung, diese ist erst mit Bestandskraft der Genehmigung/ Ermächtigung zum Betrieb einer Zweigpraxis gegeben.

Von der grundsätzlichen Überlegung, es könne sinnvoll sein, an einem weiteren Ort Leistungen anzubieten, bis zur Realisierung in Form der bestandskräftigen Genehmigung/Ermächtigung zum Betrieb der Zweigpraxis kann daher einige Zeit ins Land gehen. Es muss folglich im Zuge der Planungen einkalkuliert werden, dass sich in diesem Zeitraum die Rahmenbedingungen für die Investition in die Zweigpraxis ändern können.

\section{c) Auslagerung von Leistungen im Rahmen einer überörtlichen Berufsausübungsgemeinschaft}

Ist die Auslagerung von Leistungen in einen ausgelagerten Praxisteil beispielsweise wegen zu großer Entfernung vom Sitz der Arztpraxis nicht zulässig oder wird aus anderen Gründen für die Leistungserbringung an dem weiteren Ort die Vorhaltung einer Sprechstunde erforderlich, es bestehen jedoch aus den genannten Gründen Vorbehalte gegen einen Antrag auf Genehmigung/Ermächtigung zum Betrieb einer Zweigpraxis, so kommt eine Auslagerung von Leistungen im Rahmen einer überörtlichen Berufsausübungsgemeinschaft in Betracht.
Besteht bereits eine ÜBAG mit einem weiteren Standort, so ist die Auslagerung von Leistungen an diesen Standort zulassungsrechtlich unproblematisch. Allerdings ist darauf zu achten, dass der Arzt, der für diesen Standort zugelassen ist, über die nötige Befähigung nach der für die jeweilige Leistung geltenden Qualitätssicherungsvereinbarung verfügt und die Leistung an dem anderen Standort erst erbracht werden kann, wenn die Genehmigung nach der Qualitätssicherungsvereinbarung erteilt ist. Honorarrechtlich ist darauf zu achten, dass durch die Auslagerung von Leistungen keine Auffälligkeiten entstehen, z. B. bei Tagesprofilen.

Wird an dem Ort, an den die Leistung ausgelagert werden soll, keine Vertragsarztzulassung vorgehalten, besteht also noch keine ÜBAG, die auch diesen Ort einschließt, so muss für diesen Ort eine Vertragsarztzulassung geschaffen werden. Dies kann dadurch geschehen, dass für diesen Standort eine Sonderbedarfszulassung beantragt wird oder eine am Praxissitz vorhandene Vertragsarztzulassung an diesen Standort verlegt wird.

Eine Sonderbedarfszulassung setzt eine quantitative oder qualitative Unterversorgung an dem Standort voraus, an den die Leistung ausgelagert werden soll. Sollen an diesen Ort nur spezielle Leistungen ausgelagert werden, so wird ein Antrag auf Erteilung einer Sonderbedarfszulassung kaum Erfolg haben. Eine Sonderbedarfszulassung kann nämlich nur erteilt werden, wenn an dem Ort, für den die Sonderbedarfszulassung beantragt wird, eine Unterversorgung in der Breite des jeweiligen Fachgebiets besteht. Sollen z. B. in einer radiologischen Praxis MR-Leistungen auf diesem Wege an einen anderen Standort ausgelagert werden, so kann ein Antrag auf Erteilung einer Sonderbedarfszulassung scheitern, wenn an diesem Standort nicht auch andere Kernleistungen des Fachgebiets Radiologie angeboten werden. Anders verhält es sich jedoch, wenn an einem anderen Standort nuklearmedizinische Leistungen angeboten werden sollen, weil diese Leistungen einem eigenständigen Fachgebiet zuzurechnen sind. Bei einem Antrag auf Sonderbedarfszulassung kommt es - und dies erhöht die 
rechtlichen Schwierigkeiten dieser Auslagerungsvariante erheblich - zudem darauf an, dass an dem Ort, an dem die Leistungen erbracht werden sollen, ein quantitativer oder qualitativer Bedarf besteht. Eine Verbesserung der Versorgung der Versicherten wie bei einer Zweigpraxis reicht nicht aus. Ein Bedarf wird bspw. - anders als eine Verbesserung der Versorgung - zu verneinen sein, wenn die Leistung voraussichtlich nur von einer eher geringen Zahl von Versicherten in Anspruch genommen werden wird; in diesem Fall handelt es sich nicht um eine Unterversorgung, vielmehr ist es den Versicherten zumutbar zwecks Inanspruchnahme der Leistung eine weiter entfernte Praxis aufzusuchen.

Schließlich besteht noch die Möglichkeit der Verlegung der ganzen oder hälftigen Zulassung eines der am Sitz der Arztpraxis tätigen Vertragsärzte. Diese Auslagerungsvariante kommt jedoch für eine Einzelpraxis nicht in Betracht, da der einzelne Vertragsarzt nicht mit sich selbst eine überörtliche Berufsausübungsgemeinschaft bilden kann.

Auch bei einer am Sitz der Praxis bereits bestehenden Berufsausübungsgemeinschaft hat die Verlegung einer Zulassung Grenzen: Zum einen ist sie auf den Planungsbereich beschränkt. Zum anderen dürfen nach § 24 Abs. 7 Ärzte-ZV dem Antrag auf Verlegung der Zulassung keine Gründe der vertragsärztlichen Versorgung entgegenstehen. Zu prüfen ist dabei, ob die lokale Versorgung am bisherigen Vertragsarztsitz beeinträchtigt würde; weiterhin ist die Versorgungslage am projektierten Sitz zu beurteilen und zu prüfen, wie sich die Versorgungslage am bisherigen Vertragsarztsitz im Verhältnis zur Versorgungslage am projektierten Sitz darstellt.

Es müssen alle für die Versorgungslage maßgeblichen Aspekte - darunter Versorgungsgrad, Zahl und Ausrichtung der Praxen im Umfeld des alten Standortes, Verkehrsverbindungen u. a. - berücksichtigt werden. Bei der Feststellung, ob Gründe der Versorgung der Sitzverlegung entgegenstehen, steht den Zulassungsgremien zudem ein der gerichtlichen Kontrolle nur eingeschränkt zugänglicher Beurteilungsspielraum zu. Der Grund für die Einräumung eines solchen Spielraums liegt darin, dass nur die ortsnahen und fachkundig besetzten Zulassungsgremien sachgerecht beurteilen können, ob von der beabsichtigten Verlegung nachteilige Auswirkungen für die Versorgung der Versicherten zu besorgen sind. Auch bei dieser Auslagerungsvariante ergeben sich also aufgrund der Unvorhersehbarkeit der Entscheidung der zuständigen Zulassungsgremien, Unsicherheiten für die geplante Investition.

\section{Fazit}

Investitionsentscheidungen, die im Zusammenhang mit der Auslagerung von Leistungen im Fachgebiet Radiologie zu treffen sind, bedürfen zu ihrem Gelingen neben einer betriebswirtschaftlichen Analyse, ob sich die Investition rechnet, einer umfassenden vertragsarztrechtlichen Prüfung.

Hierbei ist zunächst zu beurteilen, wie die Auslagerung erfolgen soll, ob ein ausgelagerter Praxisteil geschaffen, eine Nebenbetriebsstätte errichtet oder ein weiterer Standort im Rahmen einer überörtlichen Berufsausübungsgemeinschaft gegründet werden soll. Je nach vertragsarztrechtlicher Gestaltung der Auslagerungsprozesse sind Anzeige- oder Genehmigungserfordernisse zu beachten. Für die Gründung einer Zweigpraxis oder eines weiteren Standorts im Rahmen einer überörtlichen Berufsausübungsgemeinschaft bestehen Investitionsrisiken. Diese ergeben sich aus unbestimmten Rechtsbegriffen der Genehmigungstatbestände, aus dem daran geknüpften Beurteilungsspielraum der Zulassungsgremien und Drittwiderspruchsrechten konkurrierender Praxen. Ferner sind Vorgaben der Qualitätssicherungsrichtlinien und honorarrechtliche
Aspekte zu berücksichtigen. Dies zwingt dazu, die vertragsarztrechtlichen Risiken vor der endgültigen Auslagerungs-/Investitionsentscheidung eingehend zu prüfen.

Die vorstehenden Überlegungen sind gleichwohl nicht nur vor der Auslagerungsbzw. Investitionsentscheidung relevant; sie sind auch nach einem erfolgreichen Auslagerungs-/Investitionsprozess von Bedeutung: Ist z. B. eine Auslagerung in einen ausgelagerten Praxisteil erfolgt und entwickelt sich dieser zu einer „verkappten“ Zweigpraxis oder nimmt die Leistungserbringung im ausgelagerten Praxisteil derart zu, dass die Gefahr besteht, dass diese die Leistungserbringung am Praxisstandort überwiegt, stellt sich die Frage, ob die Voraussetzungen für den Betrieb des ausgelagerten Praxisteils noch gegeben sind. Ist dies nicht mehr der Fall, so ergibt sich hieraus die Befugnis der zuständigen Kassenärztlichen Vereinigung zur sachlich-rechnerischen Berichtigung der für die im ausgelagerten Praxisteil erbrachten Leistungen gezahlten Vergütung - ggfs. auch rückwirkend. Es ist Aufgabe und Verantwortung des Vertragsarztes zu prüfen, ob die Voraussetzungen für den Betrieb des ausgelagerten Praxisteils noch vorliegen und ggfs. rechtzeitig den Antrag auf Genehmigung einer Zweigpraxis zu stellen oder die Leistungserbringung so zu gestalten, dass die Leistungen überwiegend am Praxissitz erbracht werden.

\section{Dr. Horst Bonvie \\ Rechtsanwalt \\ Fachanwalt für Medizinrecht}

Rechtsanwälte Wigge

Großer Burstah 42

20457 Hamburg

Telefon: (040) 3398 705-90

Telefax: (040) 3398 705-99

Internet: www.ra-wigge.de

E-Mail: kanzlei@ra-wigge.de 